Reearch Article

\title{
Elastoplastic Analysis of Tunnel Surrounding Rocks based on the Statistical Damage Constitutive Model
}

\author{
C. Q. Zhu ${ }^{1,2^{*}}$, Z. Q. Yin ${ }^{1,2}$, C. M. Li ${ }^{1,2}$ and R.M. Feng ${ }^{3}$ \\ ${ }^{I}$ Anhui Province Key Laboratory of Mining Response and Disaster Prevention and Control in Deep Coal Mine, Anhui University of \\ Science and Technology, Huainan, Anhui 232001, China \\ ${ }^{2}$ School of Resource and Safety Engineering, Anhui University of Science and Technology, Huainan, Anhui 232001, China \\ ${ }^{3}$ Department of Mining and Mineral Resources Engineering, Southern Illinois University Carbondale, Carbondale, Illinois 62901, USA
}

Received 8 May 2016; Accepted 23 June 2016

\begin{abstract}
Accurately determining the stress status of surrounding rocks is difficult as stress in surrounding rocks is redistributed after tunneling. A constitutive model selection of surrounding rocks based on the pre-peak strain hardening characteristics of surrounding rocks is considered to further analyze the mechanical characteristics of tunnel surrounding rocks. Theoretical analysis and analytic calculation were conducted on the stress distribution and the deformation characteristics of tunnel surrounding rocks in this study. The evolution laws of stress, as well as the strain of the elastoplastic zone of tunnel surrounding rocks, and the plastic zone radius along with strain hardening and other relevant factors were also analyzed. Results show that, in view of strain hardening, the mechanical characteristics of surrounding rocks increasingly approaches that of reality and the plastic zone radius achieved is larger than the result of the Kastner solution. Moreover, when the strain hardening stage is shortened, the plastic zone radius initially decreases and then slightly increases; tangential strain and displacement increase in varying degrees as the radial strain gradually decreases; tangential stress peak gradually increases and the sudden change of its distribution curve becomes increasingly evident although radial stress changes are less obvious; and sensitivity of the radius and displacement of the surrounding rock plastic zone that support resistance increase with the increase in mining depth. As a result, the research findings solve, to a certain degree, the problem that the constitutive model of surrounding rocks failed to reflect the true characteristics of surrounding rocks, providing the theoretical foundation of stability analysis and supporting the design of tunnel surrounding rocks.
\end{abstract}

Keywords: Tunnel Surrounding Rocks, Statistical Damage Constitutive Model, Strain Hardening, Elastoplastic Analysis

\section{Introduction}

After tunneling, the balanced initial triaxiality of surrounding rocks shifts to the two-dimensional stress state, which causes deformation, displacement, and even destruction of rock masses around the tunnel. Most theoretical studies on the mechanical characteristics of tunnel surrounding rocks were conducted by numerous scholars at home and abroad to assess the surrounding rock stability and to ensure an unimpeded tunnel [1],[2],[3],[4], [5],[6]. Fenner considered tunnel surrounding rocks an ideal elastoplastic medium and initially proposed an elastoplastic analysis for circular tunnel surrounding rocks, which Kastner later revised. After considering the strain softening characteristics to increase the accuracy of solutions, many scholars performed a number of improvements and fresh attempts in the treatment of the surrounding rock constitutive model[7],[8],[9],[10],[11],[12],[13],[14]. For example, Alejano et al.[7] presented a more practical elastoplastic analysis by simplifying the surrounding rock constitutive model into a linear "tri-segment" model. For the enhanced strain strengthening effect of surrounding rocks,

\footnotetext{
*E-mail address: zhuchuanqi2013@126.com

ISSN: 1791-2377 @ 2016 Eastern Macedonia and Thrace Institute of

Technology.All rights reserved.
}

Hou G.Y. et al. [8] derived an elastoplastic solution of tunnel surrounding rocks based on a power hardening constitutive model and elaborated the superiority of this model. When the constitutive relation of tunnel surrounding rocks was transformed into a "line-curve-line" model, Ranjbarnia et al. [9] analyzed stress distribution and displacement using theoretical analysis. Guo Y.H. et al. [10] used a plastic strain damage model to analyze the elastoplasticity of a circular tunnel and verified the accuracy of the analytical solution through field measurement data. However, rock has evident pre-peak strain hardening characteristics. When rock yields, the slope of the stress-strain curve decreases with the increase in stress, which decreases to 0 at the stress peak point, followed by a decrease to a negative value. The prepeak strain hardening characteristics of surrounding rocks were not highlighted in the constitutive models of surrounding rocks in previous studies, which failed to reflect the entire rock deformation process. Moreover, the internal defect of a rock should be considered in selecting the rock constitutive model because a rock is a kind of heterogeneous natural material formed after a lengthy geological process.

Therefore, selecting a model more suitable for the real constitutive relation of surrounding rocks is necessary because constitutive models of surrounding rocks hardly mirror the practical mechanical characteristics of surrounding rocks in the analysis of the elastoplasticity of tunnel surrounding rocks. According to previous studies, the 
distribution of stress and the deformation of circular tunnel surrounding rocks were analyzed in this study based on a strain hardening model. Afterward, the solution in this study was compared with the Kastner solution. Finally, the effects of strain hardening and other relevant factors on the mechanical characteristics of surrounding rocks were investigated.

The remainder of this paper is organized as follows: Part 2 describes statistical damage theory of rock in addition to the basic equations that need to be satisfied by tunnel surrounding rocks. Part 3 covers the analysis of the elastoplasticity of tunnel surrounding rocks. Part 4 compares the strain hardening with the traditional Kastner solution and contains a discussion of the effects of relevant factors on the elastoplasticity solution. Finally, the conclusions are drawn in Part 5.

\section{Primary Theories and Equations}

\subsection{Constitutive Relation of Rock based on Statistical Damage Theory}

According to statistical damage theory, the strength of any microunit inside a rock complies with the Weibull distribution, wherein the probability density function is expressed as follows [15],[16]:

$$
P(F)=\frac{m}{F_{0}}\left(\frac{F}{F_{0}}\right)^{m-1} \exp \left[-\left(\frac{F}{F_{0}}\right)^{m}\right]
$$

where $F$ acts as a distribution variable of the microunit strength random distribution and $m$ and $F_{0}$ are the Weibull distribution parameters.

Rock statistical damage variable $D$ is equal to the ratio between the already damaged microunit amount and the entire amount of the microunit. Then, the evolution equation of rock damage is expressed as follows:

$D=1-\exp \left[-\left(\frac{F}{F_{0}}\right)^{m}\right]$

The distribution variable of the microunit strength random distribution is axial strain, expressed as follows [16]:

$$
D=1-\exp \left(-\frac{\varepsilon}{\varepsilon_{c}}\right)
$$

where $\varepsilon$ is the rock strain under uniaxial compression and $\varepsilon_{\mathrm{c}}$ is the peak strain.

During rock compression, random damage to the microunit in the rock caused constant changes to the damage variable $D$. Statistical damage theory holds that the damage can be neglected when the stress is less than the rock yield strength, namely,

$$
D=0 \quad\left(\varepsilon<\varepsilon_{s}\right)
$$

where $\varepsilon_{\mathrm{s}}$ is yield strain, and the constitutive relation of rock under uniaxial compression is expressed as follows:

$$
\sigma= \begin{cases}E_{0} \varepsilon & \left(\varepsilon<\varepsilon_{s}\right) \\ E_{0} \varepsilon(1-D) & \left(\varepsilon \geq \varepsilon_{s}\right)\end{cases}
$$

where $E_{0}$ is the initial elasticity modulus, and its combination with Eq. (3) changes Eq. (5) into the following expression:

$\sigma=\left\{\begin{array}{lr}E_{0} \varepsilon & \left(\varepsilon<\varepsilon_{s}\right) \\ E_{0} \varepsilon \exp \left(-\frac{\varepsilon}{\varepsilon_{c}}\right) & \left(\varepsilon \geq \varepsilon_{s}\right)\end{array}\right.$

Fig.1shows that the rock stress-strain curve is expressed under uniaxial compression and the curve is roughly divided into three segments, namely, linear elasticity (OA segment), strain hardening (AB segment), and strain softening (BC segment). The rock statistical damage constitutive model fits the entire process curve of surrounding rock stress-strain preferably.

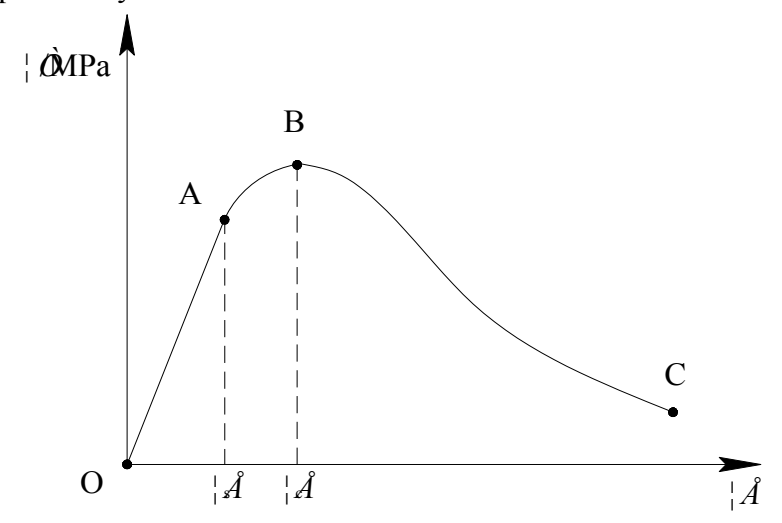

Fig.1. Rock stress-strain curve

\subsection{Mechanical Model of Circular Tunnel Surrounding Rocks}

Surrounding rock stress was redistributed after tunneling, and tunnel surrounding rocks fall into elastic and plastic zones, which later can be divided again into the plastic hardening and softening zones.

The mechanical model assumes that tunnel surrounding rocks are homogeneous, isotropic at the macrolevel, and in the equally distributed in situ rock stress field. The volume change of surrounding rocks at plastic stage is 0 , and the dead weight of surrounding rocks is neglected. A mechanical model is constructed, as shown in Fig.2, where $r_{0}$ is the tunnel radius, $p_{0}$ is the in situ rock stress, $r_{\mathrm{s}}$ is the plastic zone radius, and $p_{\mathrm{s}}$ is the support resistance.

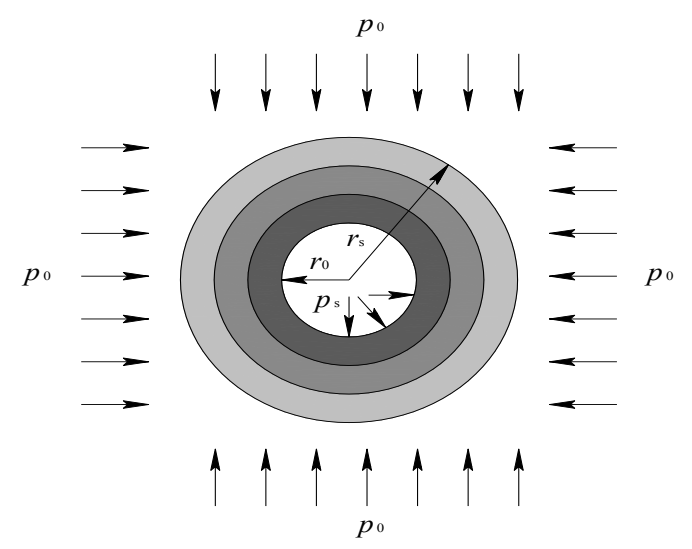

Elastic zone $\quad \square$ Plastic hardening zone

Plastic softening zone

Fig.2. Mechanical model of the circular tunnel 
The tunnel mechanical model exhibits a symmetrical plane. Afterward, the stress and strain of surrounding rocks satisfied the following equation [8]:

$$
\left.\begin{array}{l}
\varepsilon_{z}=0 \\
\varepsilon_{\theta}+\varepsilon_{r}+\varepsilon_{z}=0 \\
\sigma_{z}=\frac{\sigma_{\theta}+\sigma_{r}}{2}
\end{array}\right\}
$$

where $\varepsilon_{\theta}, \varepsilon_{\mathrm{r}}$, and $\varepsilon_{\mathrm{z}}$ are the tangential, radial, and axial strains of tunnel surrounding rocks, respectively, and $\sigma_{\theta}, \sigma_{\mathrm{r}}$, and $\sigma_{\mathrm{z}}$ are the tangential, radial, and axial stresses of tunnel surrounding rocks, respectively. As the strain components of surrounding rocks increased proportionally, and according to total strain theory[17], the constitutive relation of plastic zone surrounding rocks under complex stress can be determined through Eq. (6), which is expressed as follows:

$$
\sigma_{i}=E_{0} \varepsilon_{i} \exp \left(-\frac{\varepsilon_{i}}{\varepsilon_{c}}\right)
$$

where $\sigma_{\mathrm{i}}$ is the equivalent stress, $\varepsilon_{\mathrm{i}}$ is the equivalent strain, and $\sigma_{\mathrm{i}}$ and $\varepsilon_{\mathrm{i}}$ are expressed as follows:

$$
\left.\begin{array}{l}
\sigma_{i}=\frac{\sqrt{2}}{2} \sqrt{\left(\sigma_{\theta}-\sigma_{r}\right)^{2}+\left(\sigma_{r}-\sigma_{z}\right)^{2}+\left(\sigma_{z}-\sigma_{\theta}\right)^{2}} \\
\varepsilon_{i}=\frac{\sqrt{2}}{3} \sqrt{\left(\varepsilon_{\theta}-\varepsilon_{r}\right)^{2}+\left(\varepsilon_{r}-\varepsilon_{z}\right)^{2}+\left(\varepsilon_{z}-\varepsilon_{\theta}\right)^{2}}
\end{array}\right\}
$$

The tangential strain $\varepsilon_{\theta}$ and radial strain $\varepsilon_{\mathrm{r}}$ of surrounding rocks satisfied the following geometric equations:

$$
\left.\begin{array}{c}
\varepsilon_{\theta}=\frac{u}{r} \\
\varepsilon_{r}=\frac{d u}{d r}
\end{array}\right\}
$$

where $u$ is the radial displacement of surrounding rocks and $r$ is the distance between surrounding rocks and tunnel center.

Surrounding rocks displacement $u$ and axial strain $\varepsilon_{z}$ satisfied the following equation:

$$
\frac{d u}{d r}+\frac{u}{r}+\varepsilon_{z}=0
$$

Given that $\varepsilon_{\mathrm{z}}=0$, the expression for surrounding rock displacement $u$ was obtained. $u$ met the following equation:

$$
u=\frac{C_{1}}{r}
$$

where $C_{1}$ is the integration constant.

Tangential strain $\varepsilon_{\theta}$ and radial strain $\varepsilon_{\mathrm{r}}$ are derived from Eqs. (12) and (10). $\varepsilon_{\theta}$ and $\varepsilon_{\mathrm{r}}$ satisfied the following equations:

$$
\left.\begin{array}{l}
\varepsilon_{\theta}=\frac{C_{1}}{r^{2}} \\
\mathcal{E}_{r}=-\frac{C_{1}}{r^{2}}
\end{array}\right\}
$$

Equivalent strain $\varepsilon_{\mathrm{i}}$ is derived from the first part of Eq. (7) and the second parts of Eqs. (9) and (13). $\varepsilon_{\mathrm{i}}$ is expressed as follows:

$\varepsilon_{i}=\frac{2 \sqrt{3}}{3} \frac{C_{1}}{r^{2}}$

Eq. (14) satisfied the condition that $\varepsilon_{\mathrm{i}}=\varepsilon_{\mathrm{s}}$ when $r=r_{\mathrm{s}}$. $C_{1}$ is expressed as follows:

$C_{1}=\frac{\sqrt{3}}{2} \varepsilon_{s} r_{s}^{2}$

Surrounding rock displacement $u$, tangential strain $\varepsilon_{\theta}$, radial strain $\varepsilon_{\mathrm{r}}$, and equivalent strain $\varepsilon_{\mathrm{i}}$ are obtained from Eqs. (12) to (15). $u, \varepsilon_{\theta}, \varepsilon_{\mathrm{r}}$, and $\varepsilon_{\mathrm{i}}$ are expressed as follows:

$u=\frac{\sqrt{3}}{2} \frac{\varepsilon_{s} r_{s}^{2}}{r}$

$\varepsilon_{\theta}=\frac{\sqrt{3}}{2} \frac{\varepsilon_{s} r_{s}^{2}}{r^{2}}$

$\left.\varepsilon_{r}=-\frac{\sqrt{3}}{2} \frac{\varepsilon_{s} r_{s}^{2}}{r^{2}}\right\}$

$\varepsilon_{i}=\varepsilon_{s} \frac{r_{s}^{2}}{r^{2}}$

The constitutive relation under three-dimensional states of tunnel plastic zone surrounding rocks is derived from Eqs. (8) and (18) and expressed as follows:

$\sigma_{i}=E_{0} \varepsilon_{s} \frac{r_{s}^{2}}{r^{2}} \exp \left(-\frac{r_{s}^{2} \varepsilon_{s}}{r^{2} \varepsilon_{c}}\right) \quad\left(r_{0} \leq r \leq r_{s}\right)$

\section{Stress Distribution and Deformation Characteristics of Tunnel Surrounding Rocks}

\subsection{Elastic Zone Stress}

This study assumed that $\sigma_{\mathrm{s}}$ acts as radial stress at the tunnel surrounding rocks elastoplastic junction and supports the resistance of surrounding rocks in the plastic zone. Then, stresses of the elastic zone of tunnel surrounding rocks are expressed as follows:

$$
\left.\begin{array}{l}
\sigma_{r}=\sigma_{s} \frac{r_{s}^{2}}{r^{2}}+p_{0}\left(1-\frac{r_{s}^{2}}{r^{2}}\right) \\
\sigma_{\theta}=-\sigma_{s} \frac{r_{s}^{2}}{r^{2}}+p_{0}\left(1+\frac{r_{s}^{2}}{r^{2}}\right)
\end{array}\right\}
$$

\subsection{Plastic Zone Stress}

The expression of tangential stress $\sigma_{\theta}$ and radial stress $\sigma_{\mathrm{r}}$ of the surrounding rock plastic zone is obtained by combining the third part of Eq. (7) and the first parts of Eqs. (9) and (19). $\sigma_{\theta}$ and $\sigma_{\mathrm{r}}$ met the following equation:

$$
E_{0} \varepsilon_{s} \frac{r_{s}^{2}}{r^{2}} \exp \left(-\frac{r_{s}^{2} \varepsilon_{s}}{r^{2} \varepsilon_{c}}\right)=\frac{\sqrt{3}}{2}\left(\sigma_{\theta}-\sigma_{r}\right)
$$


Surrounding rocks stress satisfied the following equilibrium differential equation:

$$
\frac{d \sigma_{r}}{d r}+\frac{\sigma_{r}-\sigma_{\theta}}{r}=0
$$

The radial stress $\sigma_{\mathrm{r}}$ of the surrounding rock plastic zone is obtained from Eqs. (21) and (22). $\sigma_{\mathrm{r}}$ is expressed as follows:

$$
\sigma_{r}=\frac{\sqrt{3} E_{0} \varepsilon_{c}}{3} \exp \left(-\frac{r_{s}^{2} \varepsilon_{s}}{\varepsilon_{c} r^{2}}\right)+C_{2}
$$

where $C_{2}$ is the integration constant. Eq. (23) satisfied the condition that $\sigma_{\mathrm{r}}=p_{\mathrm{s}}$ when $r=r_{0}$. Moreover, the radial stress $\sigma_{\mathrm{r}}$ of surrounding rocks is expressed as follows:

$$
\sigma_{r}=\frac{\sqrt{3} E_{0} \varepsilon_{c}}{3}\left[\exp \left(-\frac{r_{s}^{2} \varepsilon_{s}}{\varepsilon_{c} r^{2}}\right)-\exp \left(-\frac{r_{s}^{2} \varepsilon_{s}}{\varepsilon_{c} r_{0}^{2}}\right)\right]+p_{s}
$$

The tangential stress $\sigma_{\theta}$ of surrounding rocks is expressed as follows:

$$
\begin{aligned}
\sigma_{\theta}= & \frac{\sqrt{3} E_{0}}{3}\left[\frac{2 r_{s}^{2} \varepsilon_{s}}{r^{2}} \exp \left(-\frac{r_{s}^{2} \varepsilon_{s}}{\varepsilon_{c} r^{2}}\right)+\varepsilon_{c} \exp \left(-\frac{r_{s}^{2} \varepsilon_{s}}{\varepsilon_{c} r^{2}}\right)\right. \\
& \left.-\varepsilon_{c} \exp \left(-\frac{r_{s}^{2} \varepsilon_{s}}{\varepsilon_{c} r_{0}{ }^{2}}\right)\right]+p_{s}
\end{aligned}
$$

\subsection{Plastic Zone Radius and Displacement}

When $r=r_{\mathrm{s}}$, the tangential stress $\sigma_{\theta}$ and radial stress $\sigma_{\mathrm{r}}$ of surrounding rocks satisfied the following expression:

$$
\sigma_{\theta}+\sigma_{r}=2 p_{0}
$$

The plastic zone radius is obtained from Eqs. (24) to (26), and $r_{\mathrm{s}}$ is expressed as follows:

$$
r_{s}=\sqrt{-\frac{\varepsilon_{c} r_{0}^{2}}{\varepsilon_{s}} \ln \left[\left(\frac{\varepsilon_{s}}{\varepsilon_{c}}+1\right) \exp \left(-\frac{\varepsilon_{s}}{\varepsilon_{c}}\right)-\frac{\sqrt{3}\left(p_{0}-p_{s}\right)}{E_{0} \varepsilon_{c}}\right]}
$$

The radial displacement $u$, tangential strain $\varepsilon_{\theta}$, and radial strain $\varepsilon_{\mathrm{r}}$ of surrounding rocks are obtained from Eqs. (16), (17), and (27). $u, \varepsilon_{\theta}$, and $\varepsilon_{\mathrm{r}}$ are expressed as follows:

$$
\begin{aligned}
& \left.\begin{array}{l}
u=-\frac{\sqrt{3}}{2} \frac{\varepsilon_{c} r_{0}^{2}}{r} \ln \left[\left(\frac{\varepsilon_{s}}{\varepsilon_{c}}+1\right) \exp \left(-\frac{\varepsilon_{s}}{\varepsilon_{c}}\right)-\frac{\sqrt{3}\left(p_{0}-p_{s}\right)}{E_{0} \varepsilon_{c}}\right] \\
\varepsilon_{\theta}=-\frac{\sqrt{3}}{2} \frac{\varepsilon_{c} r_{0}^{2}}{r^{2}} \ln \left[\left(\frac{\varepsilon_{s}}{\varepsilon_{c}}+1\right) \exp \left(-\frac{\varepsilon_{s}}{\varepsilon_{c}}\right)-\frac{\sqrt{3}\left(p_{0}-p_{s}\right)}{E_{0} \varepsilon_{c}}\right] \\
\varepsilon_{r}=\frac{\sqrt{3}}{2} \frac{\varepsilon_{c} r_{0}^{2}}{r^{2}} \ln \left[\left(\frac{\varepsilon_{s}}{\varepsilon_{c}}+1\right) \exp \left(-\frac{\varepsilon_{s}}{\varepsilon_{c}}\right)-\frac{\sqrt{3}\left(p_{0}-p_{s}\right)}{E_{0} \varepsilon_{c}}\right]
\end{array}\right\}
\end{aligned}
$$

Tunnel periphery displacement $u_{0}$ is expressed as follows:

$$
u_{0}=-\frac{\sqrt{3} \varepsilon_{c} r_{0}}{2} \ln \left[\left(\frac{\varepsilon_{s}}{\varepsilon_{c}}+1\right) \exp \left(-\frac{\varepsilon_{s}}{\varepsilon_{c}}\right)-\frac{\sqrt{3}\left(p_{0}-p_{s}\right)}{E_{0} \varepsilon_{c}}\right] .
$$

Elastoplastic analysis based on the rock statistical damage constitutive model fully considered the pre-peak hardening characteristics of surrounding rocks. The analytical expressions derived can be applied to rocks with more defects. According to the known tunnel radius $r_{0}$, in situ rock stress $p_{0}$, and support resistance $p_{\mathrm{s}}$, the field technicians can determine the plastic zone radius, stress, strain, and displacement of tunnel surrounding rocks through Eqs. (27) to (30) by simply obtaining the initial elasticity modulus $E_{0}$, yield strain $\varepsilon_{\mathrm{s}}$, and peak strain $\varepsilon_{\mathrm{c}}$. As a result, evaluating the stability and support design of tunnel surrounding rocks is easier to conduct by using the elastoplastic analysis discussed in this study.

\section{Calculation Example Comparison and Analysis}

The radius $r_{0}$ of the circular tunnel is assumed to be $3 \mathrm{~m}$, in situ rock stress $p_{0}$ of tunnel surrounding rocks is equal to 30 $\mathrm{MPa}$, and tunnel support resistance $p_{\mathrm{s}}$ is $1 \mathrm{MPa}$. The basic parameters of surrounding rocks are listed as follows: initial elasticity modulus $E_{0}=3.0 \mathrm{GPa}$, yield strain $\varepsilon_{\mathrm{s}}=12 \times 10^{-3}$, peak strain $\varepsilon_{\mathrm{c}}=36 \times 10^{-3}$, cohesion force $c=5 \mathrm{MPa}$, and angle of internal friction $\varphi=30^{\circ}$. What follows in the passage is the comparison of the solution in this study with the Kastner solution through the calculation of an example. Afterward, the effects of strain hardening and other relevant factors on the mechanical characteristics of surrounding rocks are investigated in this study.

\subsection{Results of the Comparison between Strain Hardening and Kastner Solutions}

Kastner viewed tunnel surrounding rocks as an ideal elastoplastic medium, but neglected the nonlinear deformation characteristics of surrounding rocks. Kastner solution contains difficultly obtained parameters, such as cohesion force and internal friction angle, although their analytical expressions are plain and suitable for hard surrounding rocks with less fracture.

As shown in Fig. 3, $\sigma_{\mathrm{c}}$ is the rock peak strength, $\mathrm{OA}$ is the elastic stage, $\mathrm{AB}$ is the yield stage, and rock post-peak stress is identical to rock strength.

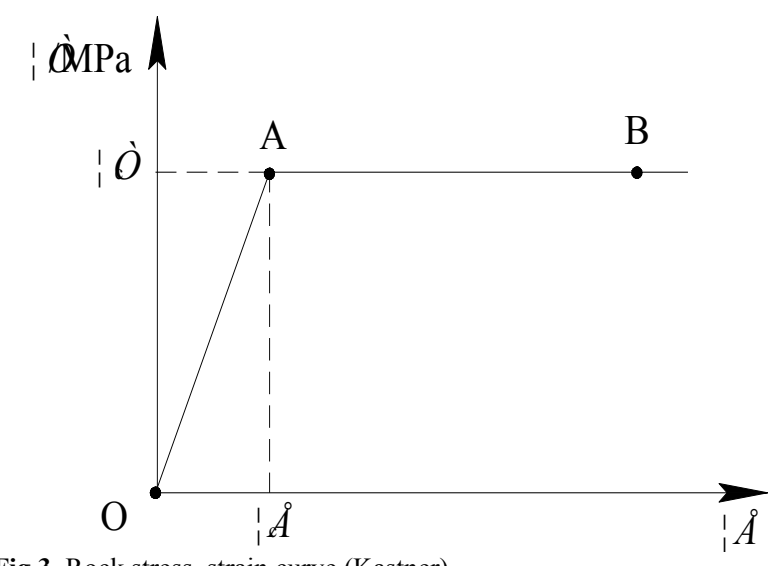

Fig.3. Rock stress-strain curve (Kastner)

The Mohr-Coulomb criterion works as a yield condition of surrounding rocks. The elastic zone stress that Kastner decided is expressed as follows: 


$$
\left.\begin{array}{l}
\sigma_{r}=p_{0}-\left(c \cos \varphi+p_{0} \sin \varphi\right) A^{\frac{1-\sin \varphi}{\sin \varphi}}\left(\frac{r_{0}}{r}\right)^{2} \\
\sigma_{\theta}=p_{0}+\left(c \cos \varphi+p_{0} \sin \varphi\right) A^{\frac{1-\sin \varphi}{\sin \varphi}}\left(\frac{r_{0}}{r}\right)^{2}
\end{array}\right\}
$$

where $A=\frac{\left(p_{0}+c \cot \varphi\right)(1-\sin \varphi)}{p_{s}+c \cot \varphi}$. Plastic zone stress is

expressed as follows:

$$
\left.\begin{array}{l}
\sigma_{r}=\left(p_{s}+c \cot \varphi\right)\left(\frac{r}{r_{0}}\right)^{\frac{2 \sin \varphi}{1-\sin \varphi}}-c \cot \varphi \\
\sigma_{\theta}=\left(p_{s}+c \cot \varphi\right)\left(\frac{1+\sin \varphi}{1-\sin \varphi}\right)\left(\frac{r}{r_{0}}\right)^{\frac{2 \sin \varphi}{1-\sin \varphi}}-c \cot \varphi
\end{array}\right\}
$$

Plastic zone radius is expressed as follows:

$$
r_{s}=r_{0} A^{\frac{1-\sin \varphi}{2 \sin \varphi}}
$$

The plastic zone radius $r_{\mathrm{s}}$ obtained in this study is $6.05 \mathrm{~m}$, whereas the plastic zone radius obtained through the Kastner solution is $4.24 \mathrm{~m}$. The distribution curves of surrounding rocks stress obtained by the solution in this study, as well as the Kastner solution, are shown in Fig. 4. Combined with Eqs. (20), (25), (31), and (32), the tangential stress obtained by the Kastner solution is a decreasing function of variable $r$ at the elastic zone and an increasing function of variable $r$ at the plastic zone. As a result, when $r=r_{\mathrm{s}}{ }^{\prime}, \sigma_{\theta}$ is maximized. By contrast, the tangential stress obtained in this study decreased with the increase in variable $r$ at the elastic zone and then increased before it decreased with the increase in variable $r$ at the plastic zone. $\sigma_{\theta}$ is not maximized when $r=r_{\mathrm{s}}$. when $r=r_{\mathrm{c}}, \sigma_{\theta}$ is maximized and $r_{\mathrm{c}}$ can be obtained through the derivation of Eq. (25). $d \sigma_{\theta} / d r$ satisfied the following equation:

$$
\frac{d \sigma_{\theta}}{d r}=\frac{2 \sqrt{3} E_{0}}{3} \exp \left(-\frac{r_{s}^{2} \varepsilon_{s}}{\varepsilon_{c} r^{2}}\right)\left(\frac{2 r_{s}^{4} \varepsilon_{s}^{2}}{\varepsilon_{c} r^{5}}-\frac{r_{s}^{2} \varepsilon_{s}}{r^{3}}\right)
$$

When $d \sigma_{\theta} / d r=0$ and $r_{\mathrm{c}}=4.94 \mathrm{~m}$.

The stress distribution laws of surrounding rocks calculated by the solution in this study and the Kastner solution basically remained the same, whereas the radial stress of surrounding rocks differed slightly in both solutions. By contrast, the tangential stress maximum obtained by the Kastner solution is observed at the elastoplastic junction, the concentration degree of tangential stress of surrounding rocks is larger, and the distribution curve of tangential stress is radically changed. The results of the solution obtained in this study are characterized by the following: the tangential stress peak of surrounding rocks is not at the elastoplastic junction, the stress distribution curve at the junction smoothly transitioned, and the stress distribution of surrounding rocks is close to the reality.

\subsection{Effects of Strain Hardening on the Elastoplastic Solution of Tunnel Surrounding Rocks}

The strain hardening stage is decided jointly by yield and peak strains. When peak strain remained certain, the effects of yield strain on the elastoplastic solution can be analyzed through $\varepsilon_{\mathrm{s}}$. Table 1 shows the calculation results of the plastic zone radius of surrounding rocks and the tunnel periphery displacement under different yield strains. Fig. 5 presents the change law of the plastic zone radius of tunnel surrounding rocks along with yield strain. Fig. 6 expresses the change law of the tunnel periphery displacement along with yield strain. Fig. 7 refers to the change laws of stress distribution of the tunnel surrounding rocks along with yield strain. Finally, Fig. 8 presents the change laws of the strain distribution of tunnel surrounding rocks along with yield strain. Analysis showed that, within $2 \times 10^{-3} \leq \varepsilon_{\mathrm{s}} \leq 20 \times 10^{-3}$ when $\varepsilon_{\mathrm{s}}<14 \times 10^{-3}$, the plastic zone radius became more sensitive to $\varepsilon_{\mathrm{s}}$, and when $\varepsilon_{\mathrm{s}} \geq 14 \times 10^{-3}$, the radius was slightly sensitive to $\varepsilon_{\mathrm{s}}$. In summary, with the increase in $\varepsilon_{\mathrm{s}}$ strain, $r_{\mathrm{S}} / r_{0}$ initially decreased and then slightly increased. $r_{\mathrm{S}} / r_{0}$ initially decreased from 3.806 to 1.652 and then increased again to 1.665 .

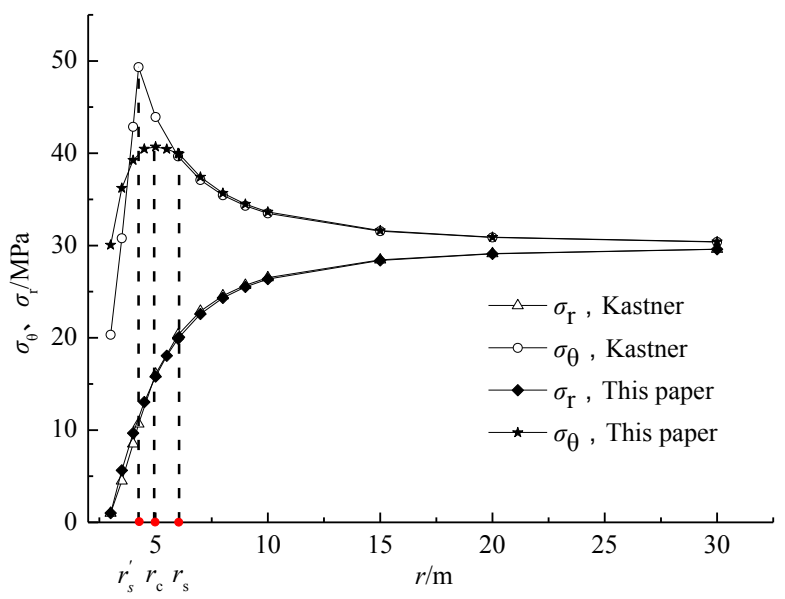

Fig.4. Stress distribution curves of tunnel surrounding rocks

\subsection{Effects of Strain Hardening on the Elastoplastic} Solution of Tunnel Surrounding Rocks

The strain hardening stage is decided jointly by yield and peak strains. When peak strain remained certain, the effects of yield strain on the elastoplastic solution can be analyzed through $\varepsilon_{\mathrm{s}}$. Table 1 shows the calculation results of the plastic zone radius of surrounding rocks and the tunnel periphery displacement under different yield strains. Fig. 5 presents the change law of the plastic zone radius of tunnel surrounding rocks along with yield strain. Fig. 6 expresses the change law of the tunnel periphery displacement along with yield strain. Fig. 7 refers to the change laws of stress distribution of the tunnel surrounding rocks along with yield strain. Finally, Fig. 8 presents the change laws of the strain distribution of tunnel surrounding rocks along with yield strain. Analysis showed that, within $2 \times 10^{-3} \leq \varepsilon_{\mathrm{s}} \leq 20 \times 10^{-3}$ when $\varepsilon_{\mathrm{s}}<14 \times 10^{-3}$, the plastic zone radius became more sensitive to $\varepsilon_{\mathrm{s}}$, and when $\varepsilon_{\mathrm{s}} \geq 14 \times 10^{-3}$, the radius was slightly sensitive to $\varepsilon_{\mathrm{s}}$. In summary, with the increase in $\varepsilon_{\mathrm{s}}$ strain, $r_{\mathrm{s}} / r_{0}$ initially decreased and then slightly increased. $r_{\mathrm{s}} / r_{0}$ initially decreased from 3.806 to 1.652 and then increased again to 1.665 .

A discrepancy in the tunnel periphery displacement was observed under different yield strains. $u_{0}$ generally increased with the increase in $\varepsilon_{\mathrm{s}}$. When $\varepsilon_{\mathrm{s}}$ increased from $2 \times 10^{-3}$ to $20 \times 10^{-3}, u_{0}$ increased from $75.257 \mathrm{~mm}$ to $144.102 \mathrm{~mm}$.

As mentioned previously, the yield strain became large, the tangential stress peak became high, and the distribution curve of tangential stress radically changed. Radial stress under various yield strains slightly changed, and when $r$ 
increased, $\sigma_{\theta}$ initially increased and then decreased and $\sigma_{\mathrm{r}}$ gradually increased.

Table 1. Plastic zone radius of surrounding rocks and tunnel periphery displacement under different $\varepsilon_{\mathrm{s}}$

\begin{tabular}{l|l|l}
\hline periphery displacement under different $\varepsilon_{\mathrm{s}}$ \\
\hline $\boldsymbol{\varepsilon}_{\mathbf{s}} \mathbf{1 0}^{-\mathbf{3}}$ & $\boldsymbol{r}_{\mathbf{s}} / \boldsymbol{r}_{\mathbf{0}}$ & $\boldsymbol{u}_{\mathbf{0}} / \mathbf{m m}$ \\
\hline 2 & 3.806 & 75.257 \\
4 & 2.725 & 77.194 \\
6 & 2.270 & 80.294 \\
8 & 2.017 & 84.531 \\
10 & 1.861 & 89.949 \\
12 & 1.761 & 96.664 \\
14 & 1.698 & 104.895 \\
16 & 1.663 & 115.016 \\
18 & 1.652 & 127.679 \\
20 & 1.665 & 144.102 \\
\hline
\end{tabular}

With the increase in $\varepsilon_{\mathrm{s}}, \varepsilon_{\theta}$ progressively increased; however, $\varepsilon_{\mathrm{r}}$ exhibited a reverse trend. By contrast, when $r$ increased, $\varepsilon_{\theta}$ gradually decreased, whereas $\varepsilon_{\mathrm{r}}$ gradually increased.

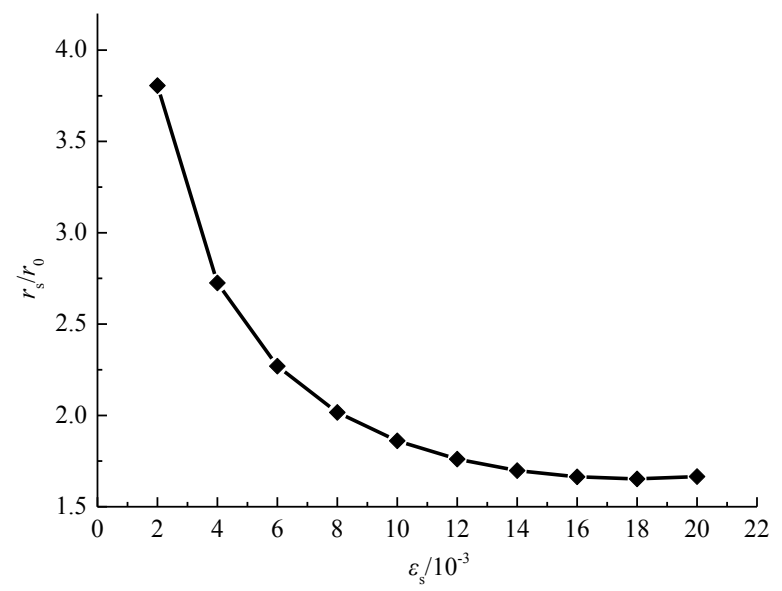

Fig.5. Change law of the plastic zone radius of tunnel surrounding rocks along with yield strain

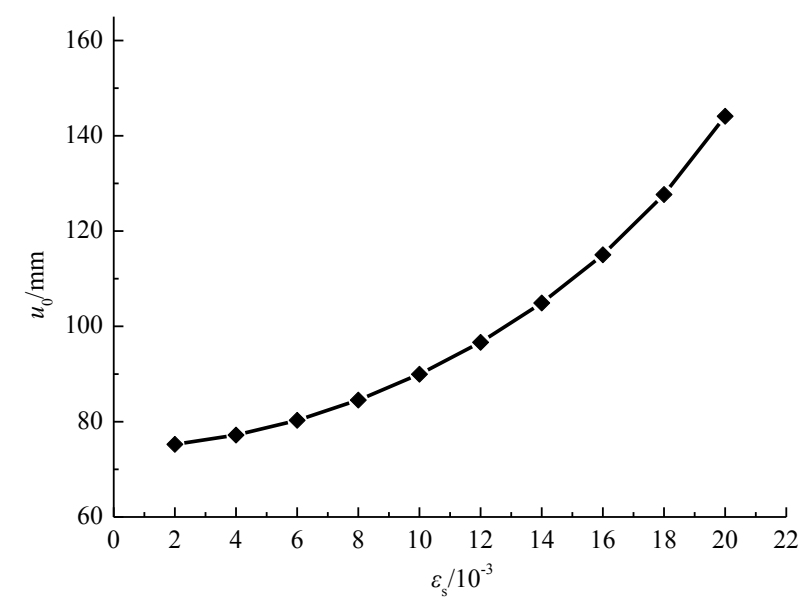

Fig.6. Change law of the tunnel periphery displacement along with yield strain

Given a larger $\varepsilon_{\mathrm{s}}$, the plastic zone radius of tunnel surrounding rocks decreased and the distribution curve of tangential stress of surrounding rocks radically changed. Therefore, the mechanical property of surrounding rocks differed largely from that of reality. When $\varepsilon_{\mathrm{s}}=\varepsilon_{\mathrm{c}}$, the constitutive relation of surrounding rocks changed into a nonlinear softening "line-curve" model. When $\varepsilon_{\mathrm{s}}=\varepsilon_{\mathrm{c}}$, the mechanical characteristics of tunnel surrounding rocks are analyzed in detail in [18].

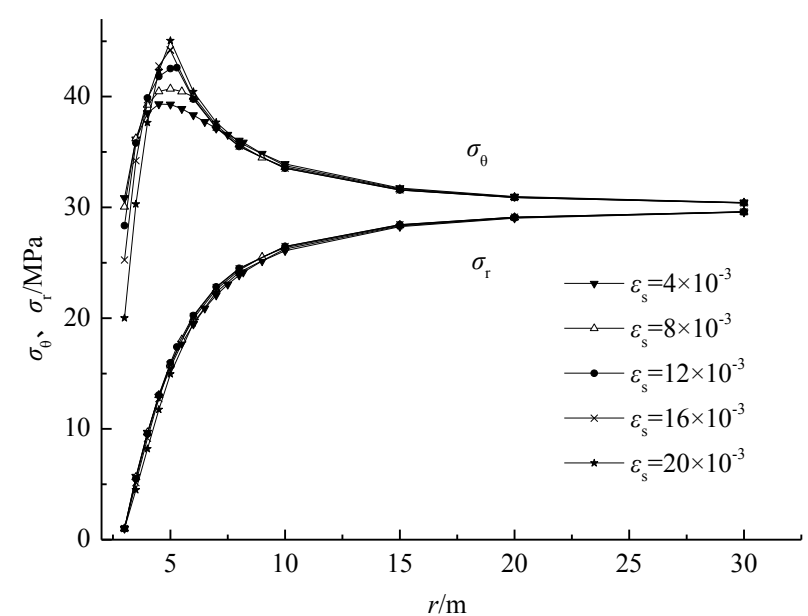

Fig.7. Change laws of the stress distribution of tunnel surrounding rocks along with yield strain

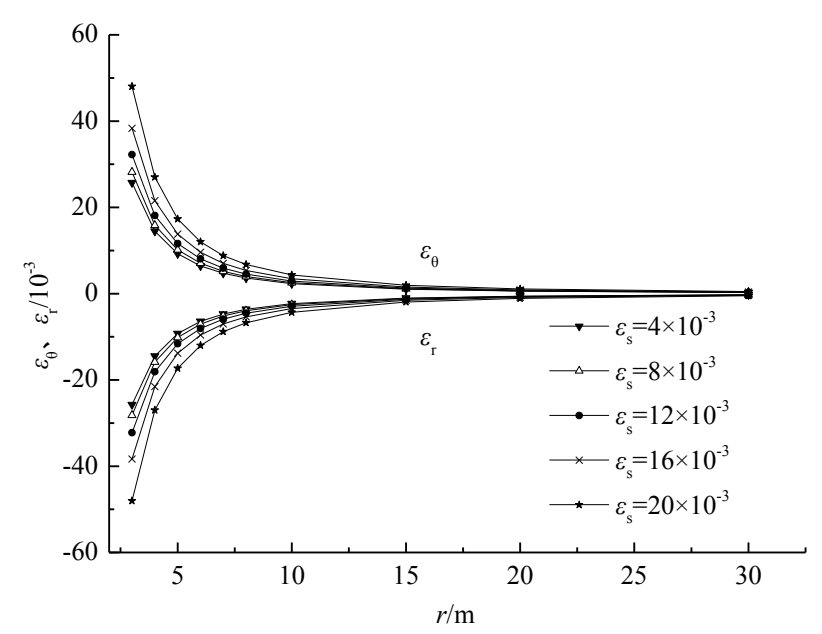

Fig.8. Change laws of the strain distribution of tunnel surrounding rocks along with yield strain

\subsection{Effects of Mining Depth and Support Resistance on the Plastic Zone Radius and Tunnel Periphery Displacement}

The tunnel surrounding rocks, which are affected by in situ rock stress and support resistance after tunneling, caused the plastic zone and displacement. Fig. 9 shows the effect laws of in situ rock stress and support resistance on the plastic zone radius of tunnel surrounding rocks. Fig. 10 presents the effect laws of in situ rock stress and support resistance on tunnel periphery displacement. Fig. 11 indicates the relation curve of in situ rock stress and plastic zone radius variations of tunnel surrounding rocks in the process of the increase in $p_{\mathrm{s}}$ from $0.5 \mathrm{MPa}$ to $4.5 \mathrm{MPa}$. Fig. 12 displays the relation curve of in situ rock stress and tunnel periphery displacement variations with the increase in $p_{\mathrm{s}}$ from $0.5 \mathrm{MPa}$ to $4.5 \mathrm{MPa}$. The results of the analysis showed that, with $p_{\mathrm{s}}$ remaining constant, when $p_{0}<35 \mathrm{MP}$, the amplifications of $r_{\mathrm{s}} / r_{0}$ and $u_{0}$ are small with the increase in $p_{0}$; when $p_{0} \geq 35 \mathrm{MPa}$, the amplifications of $r_{\mathrm{s}} / r_{0}$ and $u_{0}$ are large with the increase in $p_{0}$. When $p_{0}$ remained constant and $p_{0} \leq 35 \mathrm{MPa}, r_{\mathrm{s}} / r_{0}$ decreased by 0.20 to 0.37 and $u_{0}$ decreased by $7.75 \mathrm{~mm}$ to $35.49 \mathrm{~mm}$ with the increase in $p_{\mathrm{s}}$ from $0.5 \mathrm{MPa}$ to $4.5 \mathrm{MPa}$. When $p_{0}=40 \mathrm{MPa}, r_{\mathrm{s}} / r_{0}$ decreased by 1.35 and $u_{0}$ decreased by $185.45 \mathrm{~mm}$ with the increase in $p_{\mathrm{s}}$ from $0.5 \mathrm{MPa}$ to $4.5 \mathrm{MPa}$. Thus, the damping was extremely large. 


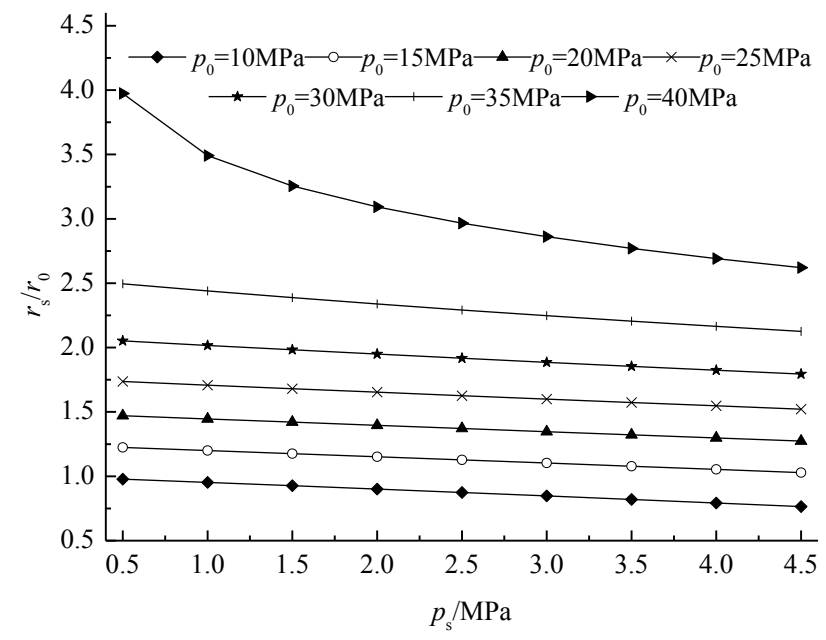

Fig.9. Effect laws of in situ rock stress and support resistance on the plastic zone radius of tunnel surrounding rocks

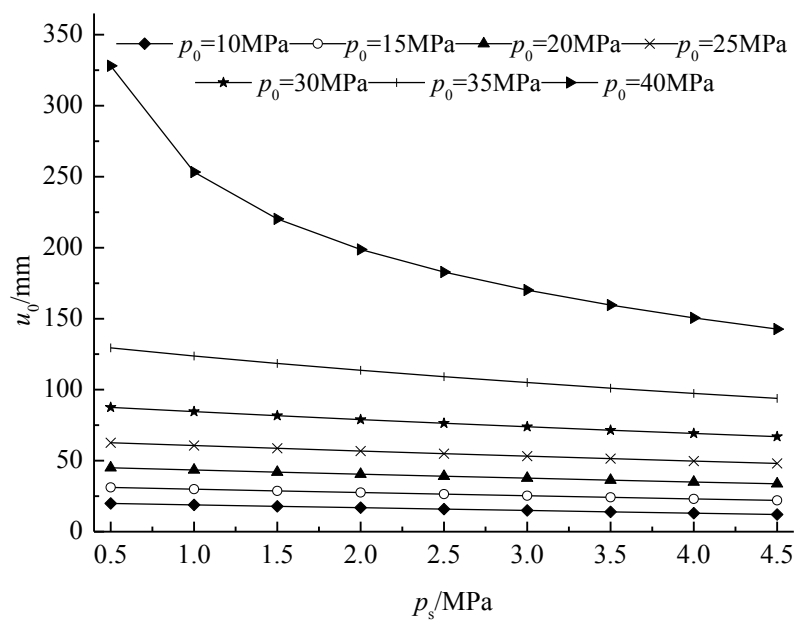

Fig.10. Effect laws of in situ rock stress and support resistance on tunnel periphery displacement

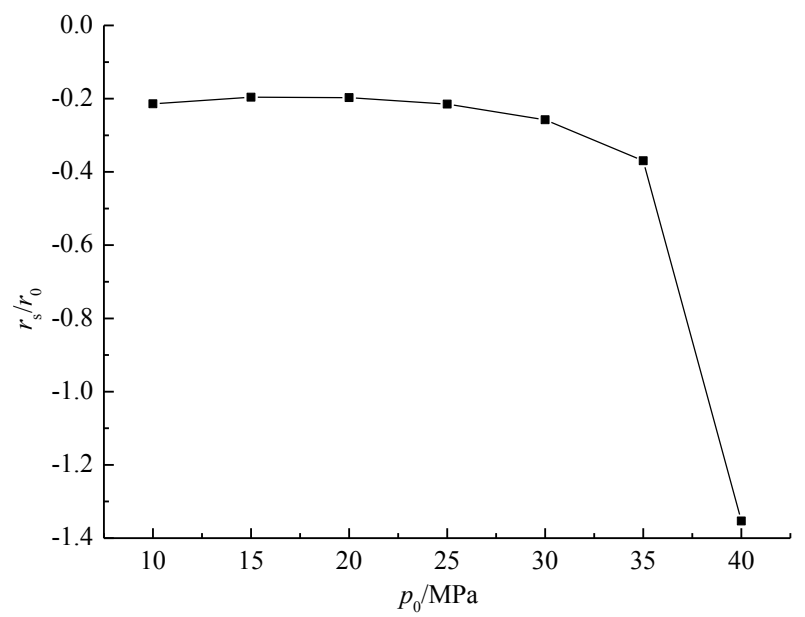

Fig.11. Relation curve of in situ rock stress and plastic zone radius variations of tunnel surrounding rocks (with the increase in $p_{\mathrm{s}}$ from 0.5 $\mathrm{MPa}$ to $4.5 \mathrm{MPa}$ )

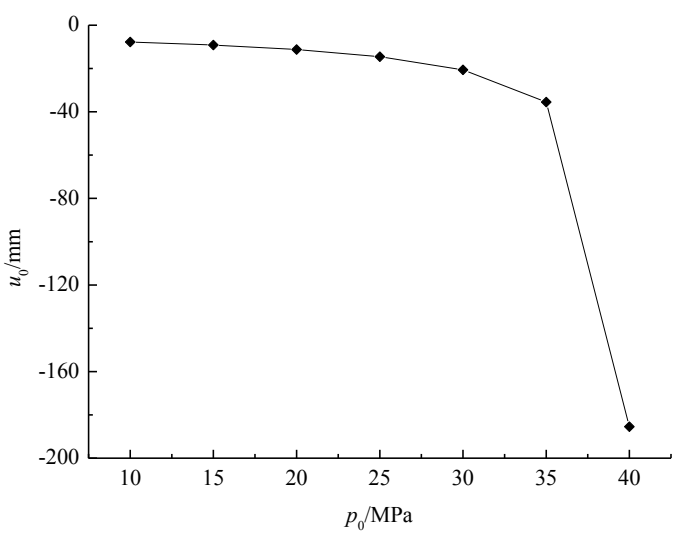

Fig.12. Relation curve of in situ rock stress and tunnel periphery displacement variations (with the increase in $p_{\mathrm{s}}$ from $0.5 \mathrm{MPa}$ to 4.5 $\mathrm{MPa})$

In situ rock stress constantly increased with the increase in mining depth. The plastic zone radius of surrounding rocks and tunnel periphery displacement continuously increased. By contrast, with the increase in support resistance, the range of the surrounding rock plastic zone and tunnel periphery displacement evidently decreases. When mining depth increases to a certain value and in situ rock stress simultaneously becomes sufficiently large, the sensitivity of the plastic zone radius and the displacement of surrounding rocks that support resistance dramatically increase. At the same moment, the advancing support resistance will largely diminish the deformation of surrounding rocks.

\section{Conclusions}

Constitutive models used in elastoplastic analysis of tunnel surrounding rocks hardly reflect the actual mechanical characteristics of surrounding rocks. The evolution law of the mechanical characteristics of tunnel periphery surrounding rocks was investigated in this study to further analyze the real deformation characteristics of surrounding rocks based on a rock statistical damage constitutive model. The following conclusions are drawn:

(1) Strain hardening is a major factor affecting stress distribution and deformation of tunnel surrounding rocks. When strain hardening occurs, the tangential stress peak of surrounding rocks fails to remain at the elastoplastic junction and the stress distribution curve becomes smooth. Furthermore, the plastic zone radius solved is larger than the result of the Kastner solution. The role that strain hardening of surrounding rocks plays cannot be overlooked in analyzing the mechanical characteristics of tunnel surrounding rocks.

(2) When mining depth increases to a certain value and when in situ rock stress is sufficiently large, the sensitivity of the plastic zone radius and the displacement of surrounding rocks that support resistance radically increase. At this moment, the advancing support resistance can largely diminish the deformation of surrounding rock deformation.

Based on rock statistical damage theory, this study explored the mechanical characteristics of the elastoplastic zone of tunnel surrounding rocks and perfected the analysis of tunnel surrounding rock elastoplasticity with increased accuracy. However, deformation at the rock plastic stage is neglected in the analysis to overcome the difficulties in 
mathematical calculation. As a result, the effect of volume changes on the plastic stage and mechanical characteristics of surrounding rocks still require further research.

\section{Acknowledgements}

This study was supported by the National Natural Science Foundation of China (Nos. 51504005 and 51304007) and the Anhui Provincial Natural Science Foundation (Nos. 1408085MKL41 and 1408085MKL42).

\section{References}

1. Fahimifar, A., Zareifard, M. R., "A new elasto-plastic solution for analysis of underwater tunnels considering strain-dependent permeability", Structure and Infrastructure Engineering, 10(11), 2014, pp.1432- 1450.

2. Gao, G.Y., Chen, Q.S., Zhang, Q.S., Chen, G.Q., "Analytical elasto-plastic solution for stress and plastic zone of surrounding rocks in cold region tunnels", Cold Regions Science and Technology, 72(2), 2012, pp.50-57.

3. Cantieni, L., Anagnostou, G., "On a paradox of elastoplastic tunnel analysis", Rock Mechanics and Rock Engineering, 44(2), 2011, pp.129-147.

4. Osman, A.S., Rouainia, M., "A semi-analytical solution for consolidation around a spherical cavity in an elasto-plastic medium and its potential application for tunneling", International Journal of Computational Methods,11(2), 2014, pp.1-13.

5. Lu, A. Z., Zhang, L. Q., Zhang, N., "Analytic stress solutions for a circular pressure tunnel at pressure and great depth including support delay", International Journal of Rock Mechanics and Mining Sciences, 48 (3),2011, pp.514-519.

6. Lu, A. Z., Xu, G. S., Sun, F., Sun, W. Q., "Elasto-plastic analysis of a circular tunnel including the effect of the axial in situ stress", International Journal of Rock Mechanics and Mining Sciences, 47(1), 2010, pp.50-59.

7. Alejano, L.R., Rodríguez-Dono, A., Veiga, M., "Plastic radii and longitudinal deformation profiles of tunnels excavated in strainsoftening rock masses", Tunnelling and Underground Space Technology, 30(4), 2012, pp.169-182.

8. Hou, G. Y., Li, J. J., Yang, Y., Wang, Y. X., Liang, Y. H., Li, Q. W., "Elastoplastic solution of axisymmetric circular tunnel based on power-hardening model", Rock and Soil Mechanics, 35(1),2014, pp.134-142.

9. Ranjbarnia, M., Fahimifar, A., Oreste, P., " Analysis of non-linear strain-softening behaviour around tunnels", Proceedings of the Institution of Civil Engineers: Geotechnical Engineering, 168(1), 2015, pp. 16-30.

10. Guo, Y. H., Jiang, F. X., Zhang, C. G., "analytical solution for critical rockburst of a circular chamber subjected to high in-situ stress", Engineering Mechanics,28(2),2011, pp.118-122.

11. Zhang, C. G., Zhao, J. H., Zhang, Q. H., Hu, X. D., “A new closedform solution for circular openings modeled by the Unified Strength Theory and radius-dependent Young's modulus", Computers and geotechnics, 42(2), 2012, pp.118- 128.

12. Fahimifar, A., Ghadami, H., Ahmadvand, M., "An elasto-plastic model for underwater tunnels considering seepage body forces and strain-softening behaviour", European Journal of Environmental and Civil Engineering, 19(2),2015, pp.129-151.

13. Wei, J. J., Jiang, B. S., Zhang, Q., "Elastic- Plastic-Brittle Unified Solution of Surrounding rocks in Deep Openings", Procedia Engineering, 26(10),2011, pp. 1225-1233.

14. Feng, Q., Jiang, B. S., Zhang, Q., "Analytical elasto-plastic solution for stress and deformation of surrounding rocks in cold region tunnels", Cold regions science and technology, 108(8), 2014, pp.59-68.

15. Krajcinovic, D., Silva, M. A. G., "Statistical aspects of the continuous damage theory", International Journal of Solids and Structures, 18(7), 1982, pp.551-562.

16. Tang, C. A., "Catastrophe in Rock Unstable Failure". Beijing: China Coal Industry Publishing House, China, pp.26-27.

17. Xu, B. Y., Chen, S. C., "Concise tutorial of plastic mechanics". Beijing: Tsinghua University Press, China, pp.84-87.

18. Pan, Y., Wang, Z. Q., "Research on relationship of loaddisplacement for cavern surrounding rocks with strain nonlinear softening", Rock and Soil Mechanics, 25(10), 2004, pp.1515-1521. 